\title{
Conference Paper \\ Synthesis of CIS Nanoink and Its Absorber Layer without Selenization
}

\section{Manoj Kumar, Raghunandan Seelaboyina, Kshitij Taneja, Alekhya Venkata Madiraju, Anup Kumar Keshri, Sarang Mahajan, and Kulvir Singh}

Centre for Nanotechnology, BHEL Corporate R\&D, Vikasnagar, Hyderabad 500093, India

Correspondence should be addressed to Manoj Kumar; manojkumar@bhelrnd.co.in

Received 1 January 2013; Accepted 3 April 2013

Academic Editors: P. Agarwal, B. Bhattacharya, U. P. Singh, and B. Sopori

This Conference Paper is based on a presentation given by Manoj Kumar at "International Conference on Solar Energy Photovoltaics" held from 19 December 2012 to 21 December 2012 in Bhubaneswar, India.

Copyright (C) 2013 Manoj Kumar et al. This is an open access article distributed under the Creative Commons Attribution License, which permits unrestricted use, distribution, and reproduction in any medium, provided the original work is properly cited.

\begin{abstract}
Highly crystalline CIS nanoink was synthesized using highly efficient microwave route. Thin films of CIS were fabricated using the developed ink by drop casting method. XRD pattern of CIS thin films indicates that a chalcopyrite phase with good crystallinity can be obtained using developed ink and that the composition of precursor ink can be transferred directly to the thin film without change in the stoichiometry. The developed ink alleviates the need of organic binders/dispersant and high temperature selenization using highly toxic $\mathrm{H}_{2} \mathrm{Se}$ gas (or $\mathrm{Na}_{2} \mathrm{Se}$ as a Se source) after deposition of thin film absorber layer. UV-VIS-NIR absorption analysis indicates that CIS thin film has a band gap of around $1.18 \mathrm{eV}$.
\end{abstract}

\section{Introduction}

Recently, commercial interest is shifted towards thin film solar cells and chalcopyrite semiconductors are expected as one of the alternative materials to $\mathrm{Si}$ solar cells. Copper indium diselenide, CuInSe ${ }_{2}$ (CIS), is a well-known ptype chalcopyrite semiconductor. CIS has direct band gap $(\sim 1.06 \mathrm{eV})$ and photon absorption coefficient of more than $10^{5} \mathrm{~cm}^{-1}$ in visible range. CIS-based solar cells have been demonstrated to have power-conversion efficiencies of more than $12 \%$ [1]. The power-conversion efficiency of the CIS absorber layer is highly dependent on the stoichiometric ratio (atomic ratios) of the elements in the layer. In recent years, nonvacuum deposition techniques with a capability to prepare large area uniform thin films with control stoichiometric composition have gained more attention [2]. Among the nonvacuum processing techniques, nanoparticle-based "inks" are considered to be simple and a cost-effective method for preparing CIS thin films. Recently, Panthani et al. [3] prepared the CIS absorber layer by using "nanocrystal ink method" in which a colloidal nanocrystal ink was obtained by reaction of $\mathrm{CuCl}, \mathrm{InCl}_{3}$, and Se in oleylamine. This approach alleviates the need for a high-temperature annealing under
Se atmosphere using highly toxic $\mathrm{H}_{2} \mathrm{Se}$ gas. In addition to this, the composition of precursor nanocrystal ink can be transferred directly to the substrates without change in the atomic ratio of elements. Films of CIS nanocrystal ink used as absorber layer in conventional-layered $\mathrm{Mo} / \mathrm{CIS} / \mathrm{CdS} / \mathrm{ZnO} / \mathrm{ITO} \mathrm{PV}$ devices exhibits reproducible photovoltaic responses with power conversion efficiencies up to $\sim 0.2 \%$. Very recently, Liu et al. [1] used a hydrazinesolution-based approach to prepare CIS absorbing layer and corresponding solar cell devices. CIS layer was prepared by dissolution of $\mathrm{Cu}_{2} \mathrm{Se}, \mathrm{In}_{2} \mathrm{Se}_{3}$, and $\mathrm{Se}$ in hydrazine solution. Complete CIS devices with glass/Mo/CIS/CdS/i-ZnO/ITO structure were fabricated by spin coating of hydrazine-based solution, which exhibit power conversion efficiencies as high as $12.2 \%$. However, in the hydrazine solution method, the toxicity of the hydrazine may cause environmental problems. In the last few decades, the solvothermal method also emerged as a powerful tool for the synthesis of nanostructures/microstructures, using organic amine as a solvent. Luo et al. [4], Jiang et al. [5], Li et al. [6], and Chun et al. [7] have reported that the CIS can be synthesized by solvothermal route, although its longer reaction time (15-36 h) and difficulty in controlling the morphology of the particles limit the 


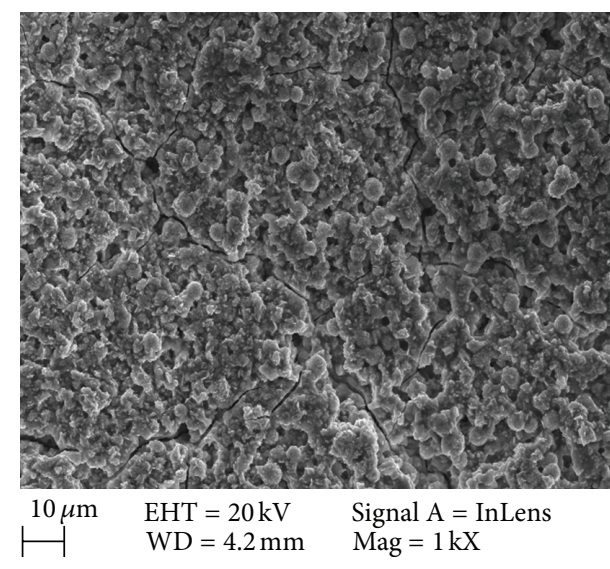

FIGURE 1: FESEM image of fabricated CIS thin film.

synthesis of high-quality CIS nanoparticles. Recently, synthesis of nanoparticles based on microwave heating is found to be a good technique because of its various advantages, such as shorter reaction time, low level of impurities, high crystallinity, excellent reproducibility, and high yield of product. Bensebaa et al. [8] used a microwave synthesis approach to prepare CIS nanoparticles. However, the use of highly toxic $\mathrm{Na}_{2} \mathrm{Se}$ (selenium source) chemicals makes this process incompatible to fabricate CIS absorber layer. The present paper describes the synthesis of highly crystalline CIS precursor ink using highly efficient microwave route. The developed ink also alleviates the need of organic binders/dispersant and high-temperature selenization using highly toxic $\mathrm{H}_{2} \mathrm{Se}$ gas or $\mathrm{Na}_{2} \mathrm{Se}$ as a Se source for deposition of thin film absorber layer.

\section{Experimental Details}

For CIS ink synthesis, the required amounts of $\mathrm{CuCl}_{2}$ $(0.205 \mathrm{~g}), \mathrm{InCl}_{3}(0.346 \mathrm{~g})$, and $\mathrm{Se}(0.248 \mathrm{~g})$ were added to $40 \mathrm{~mL}$ of ethylenediamine (ED) and mixed together. The mixture was stirred and sonicated for $30 \mathrm{~min}$, respectively, to form a homogeneous solution. Microwave synthesis was performed in a Microsynth System operating at microwave power of $600 \mathrm{~W}$ and a frequency of $2.45 \mathrm{GHz}$. The solution containing CIS precursors was sealed and heated at $180^{\circ} \mathrm{C}$ for 30 and $40 \mathrm{~min}$, respectively, for "nanoink" synthesis. The precipitate was centrifuged and washed with distilled water and absolute ethanol several times to remove byproducts. The product was filtered off and then vacuum-dried at $80^{\circ} \mathrm{C}$ for $6 \mathrm{~h}$. Phase composition and crystal structure of films are characterized by the X-ray diffraction (XRD) method. The morphology of films is observed by a field emission scanning electron microscope (FESEM). The optical absorption spectrum is recorded using UV-VIS-NIR spectrophotometer in a range of $800-1500 \mathrm{~nm}$.

\section{Results and Discussions}

The CIS thin film was fabricated using as synthesized nanoink. CIS film of thickness $\sim 1.5 \mu \mathrm{m}$ was deposited onto $25 \times 25 \mathrm{~mm}$ glass substrates by drop casting of CIS precursor ink. The procedure was repeated drop-by-drop until the

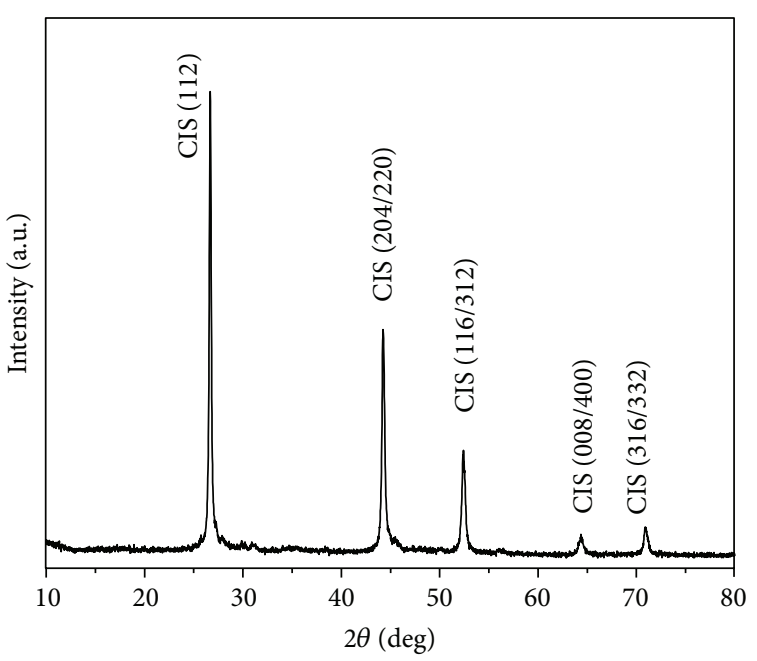

FIGURE 2: XRD pattern of CIS thin film.

sufficient thickness of CIS film is deposited. After each deposition substrate was heated to a temperature around $100^{\circ} \mathrm{C}$ to remove the solvent and form dry and smooth film with well-controlled stoichiometry among the metal compounds. This heat treatment also prevents the film from being redissolved during the next coating step. Figure 1 shows the FESEM image of the drop casting CIS thin film. The morphological investigation of CIS thin film indicates that dense and compact film having micron consists of the circular grains of size around $1-3 \mu \mathrm{m}$, which may lead to good electrical and optical properties of the absorber layer. The formation of the bigger size grains in the absorber layers is due to narrow size distribution of particles inside the ink. Smaller size particles will melt at about the same and lower sintering temperature. Due to higher surface area, nanoparticles will fuse together without any binder to form the uniform layer. In addition to this, smaller size nanoparticles will fill the voids among the bigger size particles, which results in formation of dense film having micron size grains even at temperature of around $100^{\circ} \mathrm{C}$. However, as observed from FESEM image, the drop casting method results in CIS films having cracks. These cracks could lead to undesirable shunt paths during the device fabrication. The formation of cracks can be avoided by annealing of CIS films at higher temperature under inert atmosphere. In addition to this, other suitable printing methods, like inkjet printing, could be used to make the dense and crack-free films.

Figure 2 shows the XRD pattern of CIS thin film, fabricated by drop casting of CIS nanoink synthesized for 40 min microwave irradiation time. For the irradiation time of $30 \mathrm{~min}$, many diffraction peaks appeared in the XRD pattern (not shown here), attributed to CuSe phases along with the characteristic peaks of CIS phases. As irradiation time increases from 30 to $40 \mathrm{~min}$, the secondary CuSe phase disappears and only characteristic peaks of $\mathrm{CuInSe}_{2}$ are observed, indicating the formation of pure chalcopyrite phase. XRD peaks around $2 \theta=26.6^{\circ}, 44.6^{\circ}$, and $52.8^{\circ}$, corresponding to (112), (220/204), and (116/312) planes of chalcopyrite structure. In addition to these commonly observed 


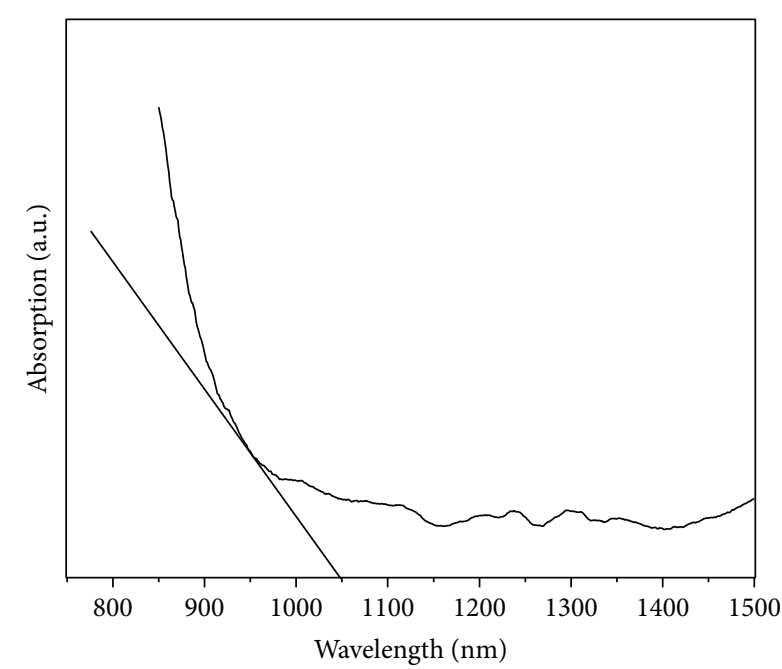

FIgURE 3: UV-VIS-NIR absorbance spectra of CIS thin film.

orientations, the weak orientations such as (008/004) and (316/332) are also observed in XRD pattern. XRD pattern of the deposited films indicates that pure chalcopyrite phase with good crystallinity was obtained using developed ink and the composition of precursor ink was transferred directly to the thin film without change in the stoichiometry of elements. Incorporation of Se in absorber layer indicates that the developed ink alleviates the need of organic binders/dispersant and high-temperature selenization using highly toxic $\mathrm{H}_{2} \mathrm{Se}$ gas (or $\mathrm{Na}_{2} \mathrm{Se}$ as a Se source) after deposition of thin film absorber layer. The observed results indicate that the Se may be incorporated into the absorber layer before the annealing stage, using $\mathrm{ED}$, because it served as both the reducing agent as well as complexing agent. A nucleophilic attack by the amines of ED activates elemental Se to form reactive $\mathrm{Se}^{2-}$ ions. The reactive $\mathrm{Se}^{2-}$ ions species react with both $\mathrm{InCl}_{3}$ and $\mathrm{Cu}^{2+}$ simultaneously in ED to form $\mathrm{In}_{2} \mathrm{Se}_{3}$ and $\mathrm{CuSe}$, respectively. Finally $\mathrm{Cu}_{2} \mathrm{Se}$ and $\mathrm{In}_{2} \mathrm{Se}_{3}$ could react, and $\mathrm{CuInSe}_{2}$ nanocrystal ink was produced. The ED avoids the need of toxic $\mathrm{Na}_{2} \mathrm{Se}$ and $\mathrm{H}_{2} \mathrm{Se}$ as a Se source, since the formation of reactive $\mathrm{Se}^{2-}$ ions species initiates the reaction (for CIS synthesis) and is easily incorporated into the absorber layer without any additional step. In order to obtain the optical property, the optical absorption spectra of fabricated film are recorded in the range of $800-1500 \mathrm{~nm}$. Figure 3 shows the UV-VIS-NIR absorbance spectra of CIS thin film fabricated by drop casting of CIS nanoink synthesized for 40 min microwave irradiation time. As seen in Figure 3, absorption begins at around $1050 \mathrm{~nm}$ wavelength. Based on this absorption behavior, the band gap of the CIS thin films can be estimated to be $1.18 \mathrm{eV}$ (obtained by extrapolating the tangent), which is in good agreement with the reported values.

\section{Conclusions}

The present paper describes the synthesis of highly crystalline CIS precursor ink using highly efficient nonvacuum microwave route. The morphological investigation of CIS thin film indicates that dense and compact film having micron size grains can be achieved using the developed ink without any selenization at higher temperature under $\mathrm{H}_{2} \mathrm{Se}$ or highly toxic $\mathrm{Na}_{2} \mathrm{Se}$ as a Se source. The developed ink also alleviates the need of organic binders/dispersant for fabrication of CIS absorber layer. X-ray diffraction pattern of the deposited films indicates that pure chalcopyrite phase with good crystallinity was obtained using developed ink and the composition of precursor ink was transferred directly to the thin film without change in the stoichiometry of elements. UV-VIS-NIR absorption analysis indicates that CIS thin film has a band gap of around $1.18 \mathrm{eV}$.

\section{References}

[1] W. Liu, D. B. Mitzi, M. Yuan, A. J. Kellock, S. J. Chey, and O. Gunawan, "12\% efficiency cuIn $(\mathrm{Se}, \mathrm{S})_{2}$ photovoltaic device prepared using a hydrazine solution process," Chemistry of Materials, vol. 22, no. 3, pp. 1010-1014, 2010.

[2] J. W. Park, Y. W. Choi, E. Lee, O. S. Joo, S. Yoon, and B. K. Min, "Synthesis of CIGS absorber layers via a paste coating," Journal of Crystal Growth, vol. 311, no. 9, pp. 2621-2625, 2009.

[3] M. G. Panthani, V. Akhavan, B. Goodfellow et al., "Synthesis of CulnS ${ }_{2}, \mathrm{CulnSe}_{2}$, and $\left.\mathrm{Cu}\left(\operatorname{In}_{x} \mathrm{Ga}_{1-x}\right)\right) \mathrm{Se}_{2}$ (CIGS) nanocrystal "inks" for printable photovoltaics," Journal of the American Chemical Society, vol. 130, no. 49, pp. 16770-16777, 2008.

[4] P. Luo, P. Yu, R. Zuo et al., "The preparation of $\mathrm{CuInSe}_{2}$ films by solvothermal route and non-vacuum spin-coating process," Physica B, vol. 405, no. 16, pp. 3294-3298, 2010.

[5] Y. Jiang, Y. Wu, X. Mo, W. Yu, Y. Xie, and Y. Qian, "Elemental solvothermal reaction to produce ternary semiconductor $\mathrm{CuInE}_{2}(\mathrm{E}=\mathrm{S}, \mathrm{Se})$ nanorods," Inorganic Chemistry, vol. 39, no. 14, pp. 2964-2965, 2000.

[6] B. Li, Y. Xie, J. Huang, and Y. Qian, "Synthesis by a solvothermal route and characterization of $\mathrm{CuInSe}_{2}$ nanowhiskers and nanoparticles," Advanced Materials, vol. 11, no. 17, pp. 1456-1459, 1999.

[7] Y. G. Chun, K. H. Kim, and K. H. Yoon, "Synthesis of CuInGaSe nanoparticles by solvothermal route," Thin Solid Films, vol. 480481, pp. 46-49, 2005.

[8] F. Bensebaa, C. Durand, A. Aouadou et al., "A new green synthesis method of $\mathrm{CuInS}_{2}$ and $\mathrm{CuInSe}_{2}$ nanoparticles and their integration into thin films," Journal of Nanoparticle Research, vol. 12, no. 5, pp. 1897-1903, 2010. 

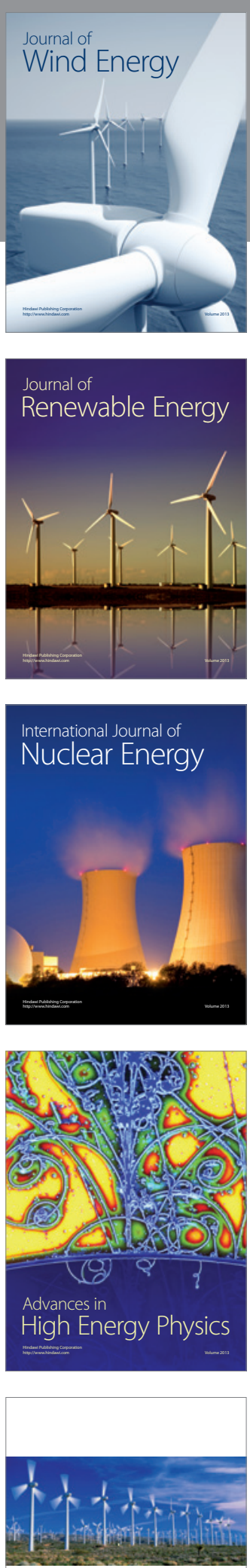

ISRN

Renewable Energy
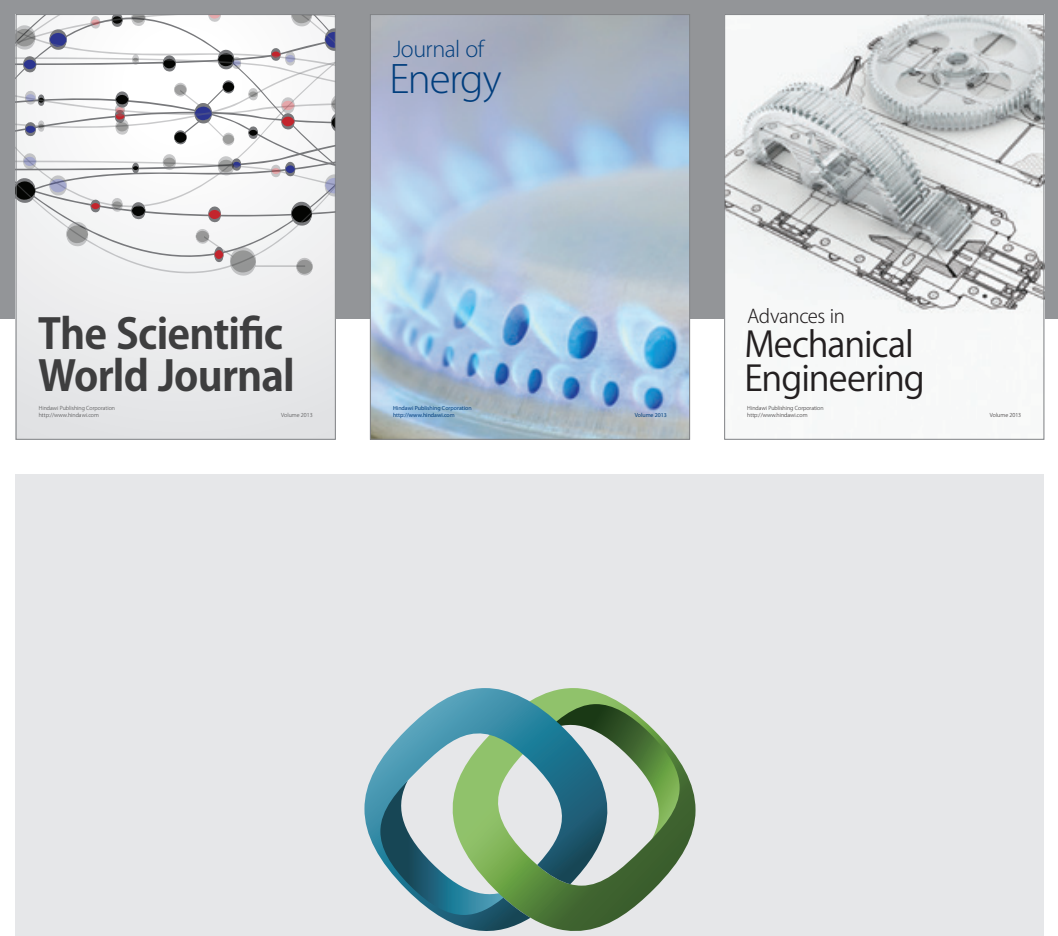

\section{Hindawi}

Submit your manuscripts at http://www.hindawi.com
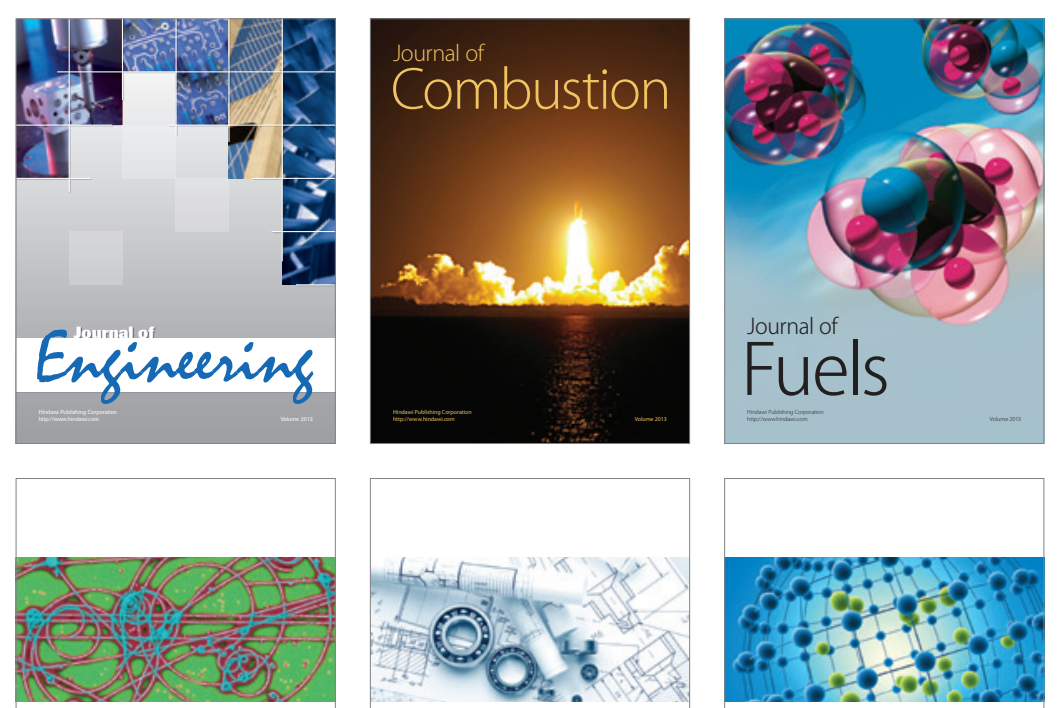

ISRN

High Energy Physics

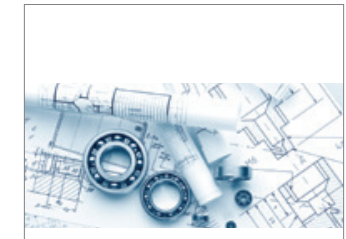

ISRN

Mechanical

Engineering

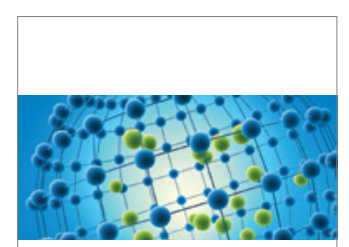

ISRN

Chemical

Engineering
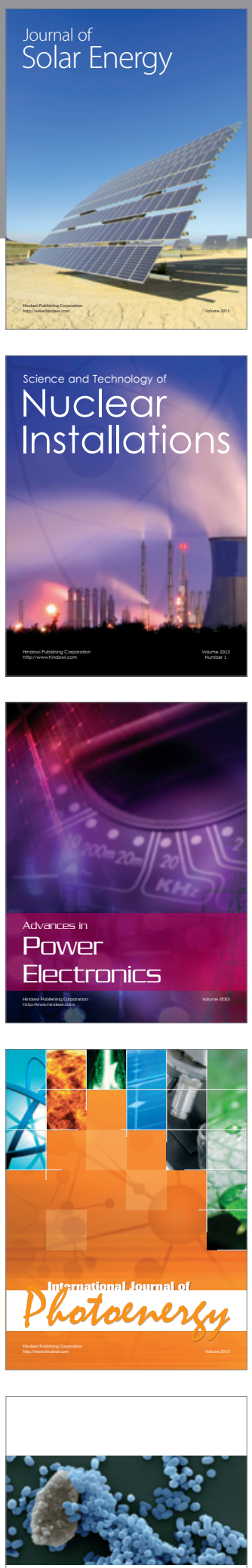

ISRN

Biotechnology 

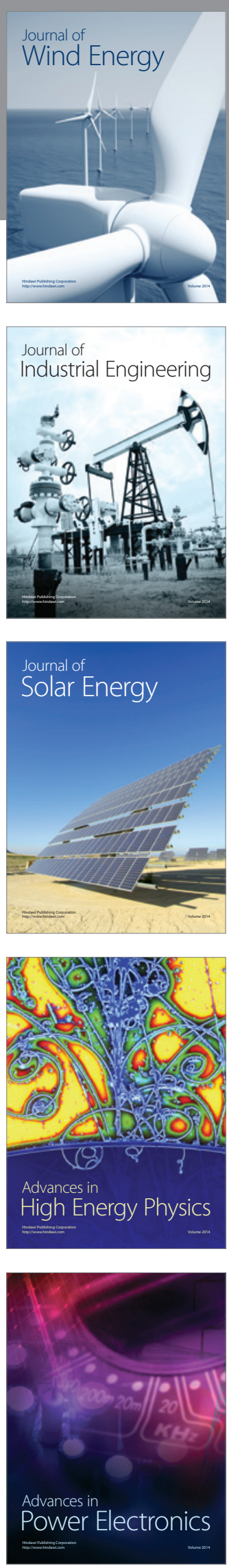
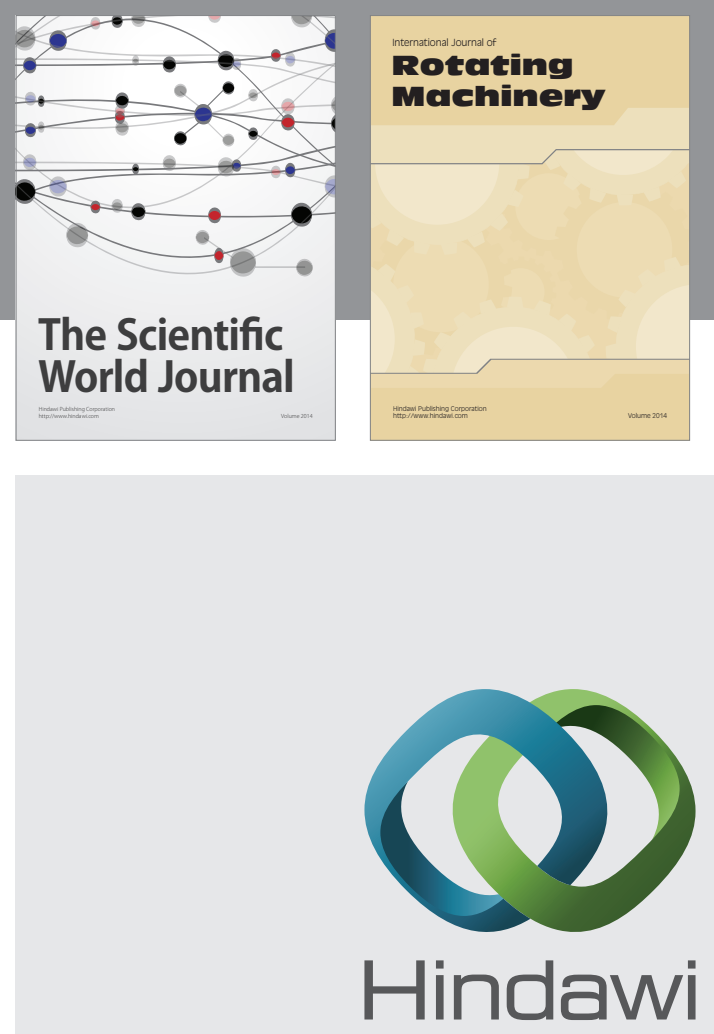

Submit your manuscripts at

http://www.hindawi.com
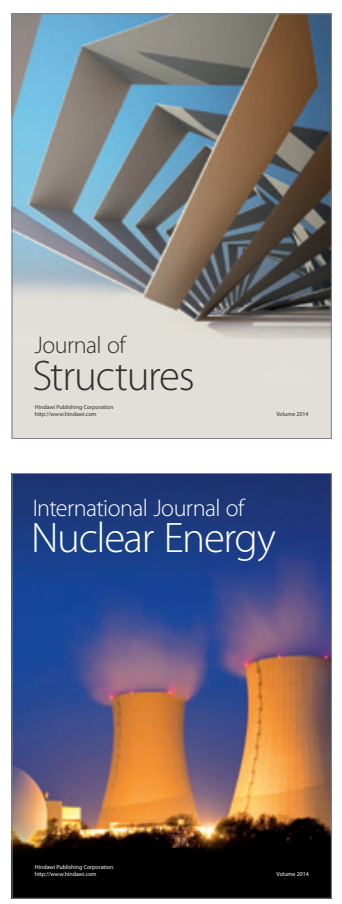
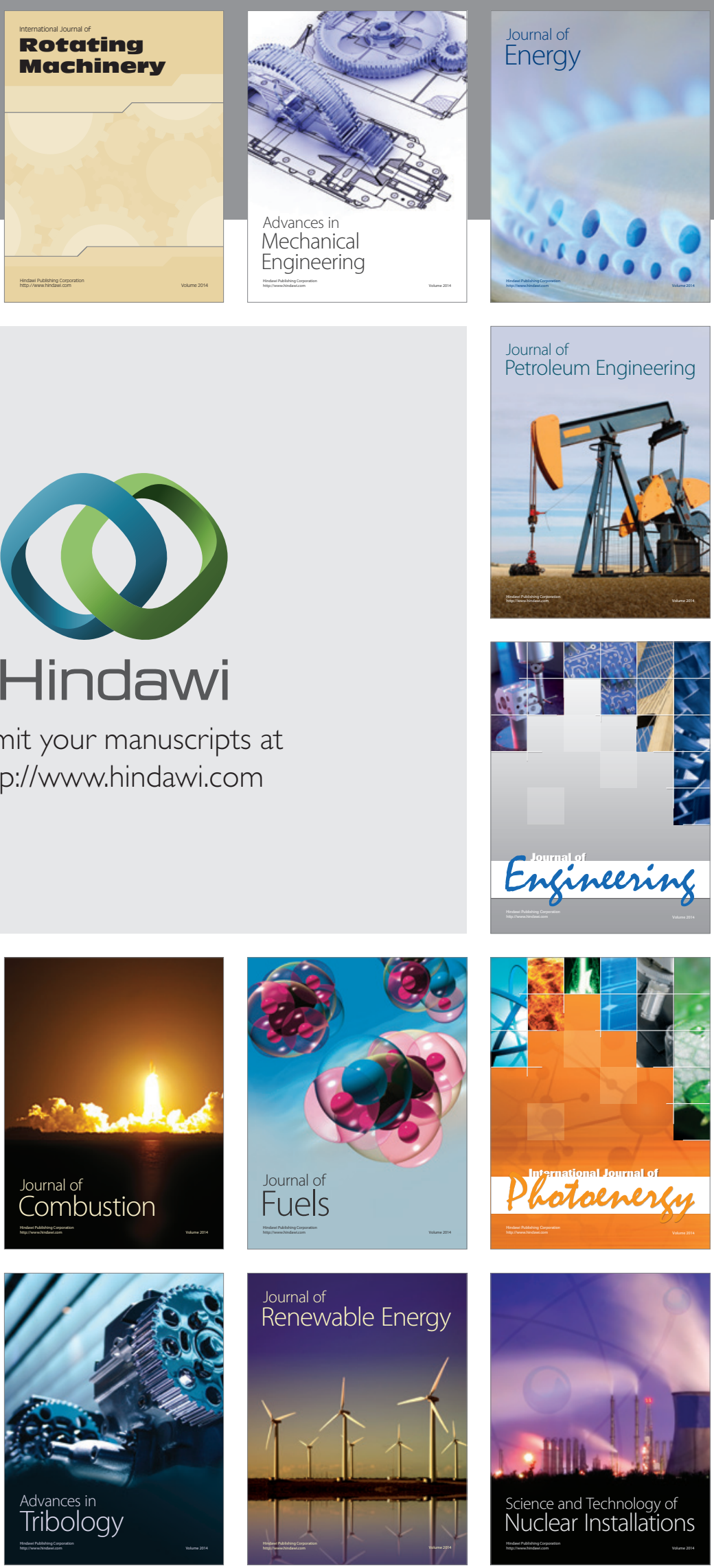